九州大学学術情報リポジトリ

Kyushu University Institutional Repository

\title{
The Number of Proofs for a BCK-Formula
}

Komori, Yuichi

Hirokawa, Sachio

Computing and Communications Center, Kyushu University

http://hdl. handle. net/2324/1546528

出版情報: The Journal of symbolic logic. 58 (2), pp.626-628, 1993-06. Association for Symbolic Logic

バージョン :

権利関係 : 


\title{
THE NUMBER OF PROOFS FOR A BCK-FORMULA
}

\author{
YUICHI KOMORI AND SACHIO HIROKAWA ${ }^{1}$
}

In this note, we give a necessary and sufficient condition for a BCK-formula to have the unique normal form proof.

We call implicational propositional formulas formulas for short. BCK-formulas are the formulas which are derivable from axioms $B=(a \rightarrow b) \rightarrow(c \rightarrow a) \rightarrow c \rightarrow b$, $C=(a \rightarrow b \rightarrow c) \rightarrow b \rightarrow a \rightarrow c$, and $K=a \rightarrow b \rightarrow a$ by substitution and modus ponens. It is known that the property of being a BCK-formula is decidable (Jaskowski [11, Theorem 6.5], Ben-Yelles [3, Chapter 3, Theorem 3.22], Komori [12, Corollary 6]). The set of BCK-formulas is identical to the set of provable formulas in the natural deduction system with the following two inference rules.

$$
\begin{gathered}
{[\gamma]} \\
\vdots \\
\gamma \rightarrow \delta
\end{gathered} \quad(\rightarrow I) \quad \frac{\gamma \rightarrow \delta \quad \gamma}{\delta} \quad(\rightarrow E) .
$$

Here $\gamma$ occurs at most once in $(\rightarrow I)$. By the formulae-as-types correspondence [10], this set is identical to the set of type-schemes of closed BCK- $\lambda$-terms. (See [5].) A BCK- $\lambda$-term is a $\lambda$-term in which no variable occurs twice. Basic notions concerning the type assignment system can be found [4]. Uniqueness of normal form proofs has been known for balanced formulas. (See $[2,14]$.) It is related to the coherence theorem in cartesian closed categories. A formula is balanced when no variable occurs more than twice in it. It was shown in [8] that the proofs of balanced formulas are BCK-proofs. Relevantly balanced formulas were defined in [9], and it was proved that such formulas have unique normal form proofs. Balanced formulas are included in the set of relevantly balanced formulas. We show a necessary and sufficient condition for a BCK-formula to have a unique

Received December 11, 1991; revised April 12, 1992.

${ }^{1}$ This work was partially supported by a Grant-in-Aid for Encouragement of Young Scientists No. 02740115 of the Ministry of Education. 
normal form proof using the following notion of minimality. The notion of BCKminimality was introduced by Komori [13]. A formula $\alpha$ is called a trivial substitution instance of $\beta$ iff $\alpha$ is a substitution instance of $\beta$ and $\beta$ is a substitution instance of $\alpha$.

Definition 1. A formula is $B C K$-minimal iff it is a BCK-formula and it is not a nontrivial substitution instance of another BCK-formula. A BCK-formula $\beta$ is a minimal formula of $\alpha$ iff $\beta$ is BCK-minimal and $\alpha$ is a substitution instance of $\beta$.

It is clear that a BCK-minimal formula is a principal type-scheme of a closed BCK- $\lambda$-term.

We identify two $\lambda$-terms when they are $\alpha$-convertible. Similarly, two types are identified when one is a trivial substitution instance of the other.

Lemma 1 ([7]). If two closed BCK- $\lambda$-terms in $\beta$-normal form have the same principal type, then they are identical.

LeMma 2 ([8]). A BCK-formula is BCK-minimal iff it is a principal type-scheme of a closed $B C K$ - $\lambda$-term in $\beta \eta$-normal form.

THEOREM 1. Given a BCK-formula $\alpha$, the number of closed $B C K$ - $\lambda$-terms in $\beta \eta-$ normal form which has type $\alpha$ is identical to the number of minimal formulas of $\alpha$.

PROOF. Let $\alpha$ be a BCK-formula. We denote by proof $(\alpha)$ the set of closed BCK$\lambda$-terms in $\beta \eta$-normal form which have type $\alpha$ and we denote by $\min (\alpha)$ the set of minimal formulas of $\alpha$. We define a function from $\operatorname{proof}(\alpha)$ to $\min (\alpha)$ and show that it is surjective and injective. Let $M \in \operatorname{proof}(\alpha)$. Then $M$ has type $\alpha$. By the principal type-scheme theorem (Theorem 15.26 of [4]), $M$ has a principal typescheme. We denote it by $\operatorname{pts}(M)$. Since $M$ is in $\beta \eta$-normal form, $\operatorname{pts}(M)$ is minimal by Lemma 2 . So we have $\operatorname{pts}(M) \in \min (\alpha)$. Thus pts is a function from proof $(\alpha)$ to $\min (\alpha)$. Injectivity of pts is immediate from Lemma 1. To prove the surjectivity, let $\beta \in \min (\alpha)$ and apply Lemma 2 to $\beta$. Then there is a closed BCK- $\lambda$-term $N$ in $\beta \eta$-normal form whose principal type-scheme is $\beta$. Therefore pts is surjective.

One consequence of the theorem is that a BCK-formula $\alpha$ has only a finite number of normal form proofs. In fact, we can enumerate all the minimal formulas instead of $\lambda$-terms. Given a formula $\gamma$, we denote by $s_{0}(\gamma)$ the set of formulas $\beta$ such that $\gamma$ is a substitution instance of $\beta$. Since we identify trivial substitution instances, the set $s_{0}(\gamma)$ is finite. Next we denote by $s(\gamma)$ the set of BCK-formulas in $s_{0}(\gamma)$. Since BCK-provability is decidable, we can enumerate the elements of $s(\gamma)$ from $s_{0}(\gamma)$. Finally note that $\beta$ is BCK-minimal iff $s(\beta)=\{\beta\}$. Therefore we have

$$
\min (\alpha)=\left\{\beta \in s_{0}(\alpha) \mid s(\beta)=\{\beta\}\right\} .
$$

Thus we can enumerate all the elements of $\min (\alpha)$.

Akama [1] showed that the number of cut-free proof (in sequent calculus) for a BCK-formula is finite.

COROLLARY 1. A BCK-formula has a unique proof in $\beta \eta$-normal form iff it has a unique minimal formula.

\section{REFERENCES}

[1] Y. AKAMA, The number of proofs and tree automaton. II, manuscript, Oct. 1991.

[2] A. A. BABAEv and S. V. Soloviev, A coherence theorem for canonical morphism in cartesian closed categories, Zapiski nauchnykh Seminarov Lenigradskogo Otdeleniya matematichskogo Instituta imeni V. A. Steklova Akademii Nauk SSSR, vol. 88 (1979), pp. 3-29. 
[3] C.'B. Ben-Yelles, Type-assignment in the lambda-calculus, Ph.D. thesis, University College, Swansea, 1979.

[4] J. R. Hindey and J. P. SELdin, Introduction to Combinators and Lambda-Calculus, Cambridge University Press, London, 1986.

[5] J. R. HindLEY, BCK-combinators and linear lambda-terms have types, Theoretical Computer Science, vol. 64 (1989), pp. 97-105.

[6] - BCK and BCI logics, condensed detachment and the 2-property, Notre Dame Journal of Formal Logic, to appear.

[7] S. HIRoKawa, Principal types of BCK-lambda terms, Theoretical Computer Science, (to appear).

[8] - Balanced formulas, BCK-minimal formulas and their proofs, Proceedings of Symposium on Logical Foundations of Computer Science, (Nerode and Taitslin, editors), Lecture Notes in Computer Science, vol. 620, Springer-Verlag, Berlin, and New York, 1992, pp. 198-208.

[9] — , BCK-formulas having unique proofs, Proceedings of category theory and computer science (Pitt et al., editors), Lecture Notes in Computer Science, vol. 530, Springer-Verlag, Berlin and New York, 1991, pp. 106-120.

[10] W. A. Howard, The formulae-as-types notion of construction, To H. B. Curry, Essays on Combinatory Logic, Lambda Calculus and Formalism (Hindley and Seldin, editors), Academic Press, Cambridge, Massachusetts, 1980, pp. 479-490.

[11] S. JASKOWSKI, Uber Tautologien, in welchen keine Variable merh Als zweimal vorkommt, Zeitschrift für Mathematische Logik und Grundlagen der Mathematik, vol. 9 (1963), pp. 219-228.

[12] Y. KомоRI, The class of BCC-algebras is not a variety, Mathematica Japonica, vol. 29 (1984), pp. $391-394$.

[13] - BCK algebras and lambda calculus, Proceedings of the 10th Symposium on Semigroups, Sakado 1986, Josai University, Sakado, 1987, pp. 5-11.

[14] G. E. MiNTs, $A$ simple proof of the coherence theorem for cartesian closed categories, manuscript, 1982.

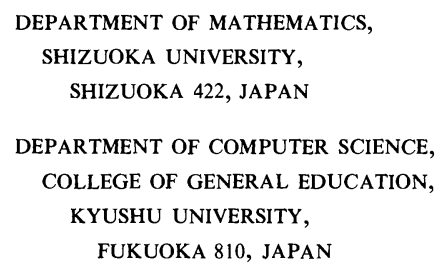

E-mail: hirokawa@ec.kyushu-u.ac.jp 